University of Nebraska - Lincoln

DigitalCommons@University of Nebraska - Lincoln

8-1981

\title{
Inhibition of In vitro Macrophage Digestion Capacity by Infection with Leishmania donovani (Protozoa: Kinetoplastida)
}

Gerald F. Kutish

Plum Island Animal Disease Center, United States Department of Agriculture

John J. Janovy Jr.

University of Nebraska - Lincoln, jjanovy1@unl.edu

Follow this and additional works at: https://digitalcommons.unl.edu/bioscijanovy

Part of the Parasitology Commons

Kutish, Gerald F. and Janovy, John J. Jr., "Inhibition of In vitro Macrophage Digestion Capacity by Infection with Leishmania donovani (Protozoa: Kinetoplastida)" (1981). John Janovy Publications. 30.

https://digitalcommons.unl.edu/bioscijanovy/30

This Article is brought to you for free and open access by the Papers in the Biological Sciences at DigitalCommons@University of Nebraska - Lincoln. It has been accepted for inclusion in John Janovy Publications by an authorized administrator of DigitalCommons@University of Nebraska - Lincoln. 


\title{
THE JOURNAL OF PARASITOLOGY
}

VOLUME 67

AUGUST 1981

NUMBER 4

J. Parasitol., 67(4), 1981, pp. 457-462

(C) American Society of Parasitologists 1981

\section{INHIBITION OF IN VITRO MACROPHAGE DIGESTION CAPACITY BY INFECTION WITH LEISHMANIA DONOVANI (PROTOZOA: KINETOPLASTIDA)}

\author{
Gerald F. Kutish and J. Janovy, Jr. \\ School of Life Sciences, University of Nebraska, Lincoln, Nebraska 68588
}

\begin{abstract}
Promastigotes of Leishmania donovani transformed into amastigotes and survived in cultured macrophages. Promastigotes of Leptomonas costoris, a kinetoplastid parasite of water striders, transformed into amastigotes but did not survive in cultured macrophages. A significant difference in size between the two species of parasitic protozoa allowed development of a bioassay in which the in vitro digestion rate of $L$. costoris was taken as a measure of macrophage digestive capacity following preinfection with $L$. donovani. In a 12- to 24-hr period, macrophage cultures infected with the $2 \mathrm{~S}$ strain of $L$. donovani retained $50 \%$ more L. costoris than did control macrophages infected with L. costoris alone. The $2 \mathrm{~S}$ strain has been in culture since 1967 and has retained its infectivity for hamsters, although its virulence is attenuated. In similar experiments with promastigotes of the Khartoum strain of $L$. donovani, a strain that evidently has lost its infectivity for hamsters, no such suppression of macrophage ability to digest L. costoris was observed. The results suggest that infective strains of Leishmania donovani suppress macrophage function beyond the time at which initial lysosomal fusion with the parasitophorous vacuole occurs.
\end{abstract}

Leishmania donovani (Protozoa: Kinetoplastida: Trypanosomatidae) is the causative agent of a variety of human diseases generally termed "visceral leishmaniasis." Other members of the genus Leishmania cause a variety of clinical infections (some self-healing and some nonhealing) of the skin and mucous membranes. Leishmania species infective to mammals are obligate intracellular parasites in the vertebrate host, although they live extracellularly as flagellated forms in the insect vector. Leishmania donovani is primarily a parasite of the reticuloendothelial system, and in vitro infected cell culture systems usually have used hamster peritoneal macrophages as the host cell (Chang and Dwyer, 1978). The exact mechanism by which these protozoa escape the macrophage killing function and, in fact, convert the macrophage into a cell supportive of parasite growth, is not known (Janovy, 1977). However, a number of recent studies has suggested that the parasite resides within a vacuole of host-cell origin, and that lysosomes do fuse with this parasitophorous vacuole soon after infection occurs (Alexander

Received 29 October 1979; revised 10 July 1980; accepted 5 November 1980. and Vickerman, 1975; Chang and Dwyer, 1976 ; 1978). Because the parasites survive lysosomal fusion, it has been suggested that they are resistant to lysosomal enzymes (Chang and Dwyer, 1976). The purpose of the present study was to examine macrophagekilling and -digestion capacity following initial infection by L. donovani. A bioassay for macrophage-killing capacity against these protozoa was developed and used to compare the effects of two strains of $L$. donovani that differ in their ability to infect hamsters.

\section{MATERIALS AND METHODS}

\section{Parasites}

Leishmania donovani, the Khartoum and $2 \mathrm{~S}$ strains (Stauber, 1966), and Leptomonas costoris, an intestinal parasite of water striders (genus Gerris), described by Wallace et al. (1965) were used. Both L. donovani strains were obtained as primary isolate (hamster), cultured flagellates from the late Dr. L. A. Stauber, Rutgers University, in 1967, and have been maintained by weekly transfers by loop in Tanabe's medium (Janovy, 1967). Leptomonas costoris was obtained from Dr. F. G. Wallace, University of Minnesota, in 1973, and has been maintained by weekly transfer by loop in Mansour's medium (Dollahon and Janovy, 1971). Leptomonas costoris has proven noninfective for rodents (Daggett et al., 1978). Parasites used to infect macro- 
phages were harvested by centrifugation $(500 \mathrm{~g}$ at $25 \mathrm{C}$ for $12 \mathrm{~min}$ ) from late log phase cultures after $1 \mathrm{wk}$ of growth, washed once in Dulbecco's phosphate-buffered saline (PBS), and diluted in macrophage cell culture medium (CCM) consisting of the following: Medium 199 (GIBCO), 15\% v/v heat-inactivated fetal calf serum (GIBCO), $0.1 \%$ lactalbumin hydrolysate (DIFCO) with $25 \mathrm{mM}$ HEPES (Sigma), and $10 \mathrm{mM}$ bicarbonate buffers. Cell viability as checked by Erythrosin B dye-exclusion was more than $99 \%$ both before and after harvest of parasites.

The two L. donovani strains were chosen for their differences in infectivity for hamsters. The $2 \mathrm{~S}$ strain will kill hamsters in 7 to 9 mo when injected intracardially as a $10^{6} / \mathrm{ml}$ suspension of promastigotes (flagellate). Approximately $10^{5}$ flagellates were given in $0.1 \mathrm{ml}$ PBS. The amastigotes recovered from passage through one animal will kill hamsters in 1.5 to 3 mo when injected as an amastigote suspension in homogenized, infected, hamster spleen. The Khartoum (Kh) strain evidently has lost its infectivity in culture. Our repeated attempts to recover parasites from hamsters have failed, even as early as $24 \mathrm{hr}$ postinoculation.

\section{Host cells}

Resident, normal, unelecited macrophages were used in all experiments and were harvested by the method of Wasley and John (1972). Two-month-old male Syrian Golden hamsters (Mesocricetus aurotus) (90 g weight) from a random outbred strain maintained at the University of Nebraska-Lincoln Life Science Animal Facility were killed with chloroform. Approximately $15 \mathrm{ml}$ cold, heparinized (10 $\mathrm{IU} / \mathrm{ml}) \mathrm{CCM}$ was injected into the peritoneal cavity and withdrawn after $3 \mathrm{~min}$. The peritoneal cell suspension was seeded at $1.2 \times 10^{6}$ cells in $1 \mathrm{ml}$ per Leighton tube with glass coverslips $(9 \times 35 \mathrm{~mm}$, Bellco) and incubated at $35 \mathrm{C}$ with $95 \%$ air $-5 \% \mathrm{CO}_{2}$. To enhance cell attachment, coverslips were coated with $0.5 \%$ aqueous poly-L-lysine, MW 70,000 (Sigma Type I-B), rinsed, and steam sterilized in the Leighton tubes. Nonadherent cells were aspirated after $1 \mathrm{hr}$ while the remaining adherent macrophages (at approximately $1.5 \times 10^{5} / \mathrm{cm}^{2}$ ) were incubated in CCM without heparin and used immediately.

\section{Bioassay}

A bioassay to determine if $L$. donovani altered macrophage capacity to digest $L$. costoris was performed in the following ways: (1) a sequential infection using an initial infection with $L$. donovani followed by a subsequent infection with $L$. costoris, and (2) a simultaneous infection with both $L$. donovani and L. costoris.

For bioassay by sequential infection, the peritoneal macrophages were exposed to $L$. donovani, $2 \mathrm{~S}$ or Kh strains, at a ratio of two parasites/adherent macrophage for $4 \mathrm{hr}$ at $35 \mathrm{C}$ in $95 \%$ air $-5 \% \mathrm{CO}_{2}$. After vigorously washing twice in PBS to remove noningested parasites, the macrophages were ex- posed to $L$. costoris at a ratio of one parasite/adherent macrophage, again vigorously washed twice in PBS to remove noningested parasites (according to the methods of Chang and Dwyer, 1976), and reincubated. Macrophages in the control were exposed for $4 \mathrm{hr}$ to L. costoris alone.

For bioassay by simultaneous infection, the peritoneal macrophages were exposed to $10^{6} \mathrm{~L}$. donovani $2 \mathrm{~S}$ and $10^{6} \mathrm{~L}$. costoris for $4 \mathrm{hr}$ at $35 \mathrm{C}$. Control macrophages were exposed for $4 \mathrm{hr}$ to (1) $10^{6} \mathrm{~L}$. costoris alone, (2) $10^{6}$ heat-killed $2 \mathrm{~S}(56 \mathrm{C}, 30 \mathrm{~min}$ ) with $10^{6}$ L. costoris, and (3) $10^{6}$ heat-killed Kh with $10^{6}$ L. costoris. Following infection, the macrophages were washed vigorously to remove noningested parasites and then reincubated.

The number of $L$. costoris remaining in the host cells at various times following ingestion was used as an assessment of the macrophage's digestive capacity. Leptomonas costoris was distinguishable from $L$. donovani, even when partially digested within a macrophage vacuole. The following Giemsa-stained attributes of the parasite were used: nuclear size, nucleolus, body size, cytoplasmic granules, and kinetoplast. Leptomonas costoris (9-11 $\mu \mathrm{m}$ diameter) was always two to three times larger than $L$. donovani $(2.5-4.0 \mu \mathrm{m})$ and contained distinct cytoplasmic granules (Fig. 1). Upon initial phagocytosis, $L$. costoris had an eosinophilic nucleus (3-4 $\mu \mathrm{m}$ diameter) with a very large nucleolus $(2-3 \mu \mathrm{m})$, and dense azurophilic granules with a lightly blue staining cytoplasm. Partial digestion within a vacuole was apparent as evidenced by translucent and nonstaining cytoplasm. Continued digestion was recognized by the presence of clear cytoplasm, azurophilic granules, but no nucleus or kinetoplast. Leptomonas costoris was identified based on the presence of the cytoplasmic granules. Vacuoles with obvious cytoplasmic bodies two to three times larger than $L$. donovani and without nucleus, kinetoplast, or cytoplasmic granules were not counted.

Leishmania donovani within a macrophage vacuole had an eosinophilic nucleus (1.8 $\mu$ m diameter) and an azurophilic cell body $(2.5 \mu \mathrm{m}$ diameter). Parasites that were digested partially had a pycnotic nucleus ( $1 \mu \mathrm{m}$ diameter) and an unstained translucent cell body ( $4 \mu \mathrm{m}$ diameter). Leishmania donovani was identified based on the presence of both a nucleus and a kinetoplast. Vacuoles with an obvious cytoplasmic body but no nucleus or kinetoplast were not counted.

Coverslips were removed at various times, airdried, fixed in methanol, and stained with JennerGiemsa. Treatments were coded, and parasites on coverslips counted without knowledge of experimental treatment using a stratified random sampling to estimate the parasite burden (parasites/ macrophage). From 200 to 600 macrophages per replicate were counted to estimate the parasite burden with $95 \%$ probability of having an error less than $5 \%$. Replicate samples were tested for homogeneity (chi-square) and pooled. Results were expressed as parasites per macrophage at a given time following infection and as percent change in parasite burden during a given time interval. A chisquare contingency table analysis was used to 


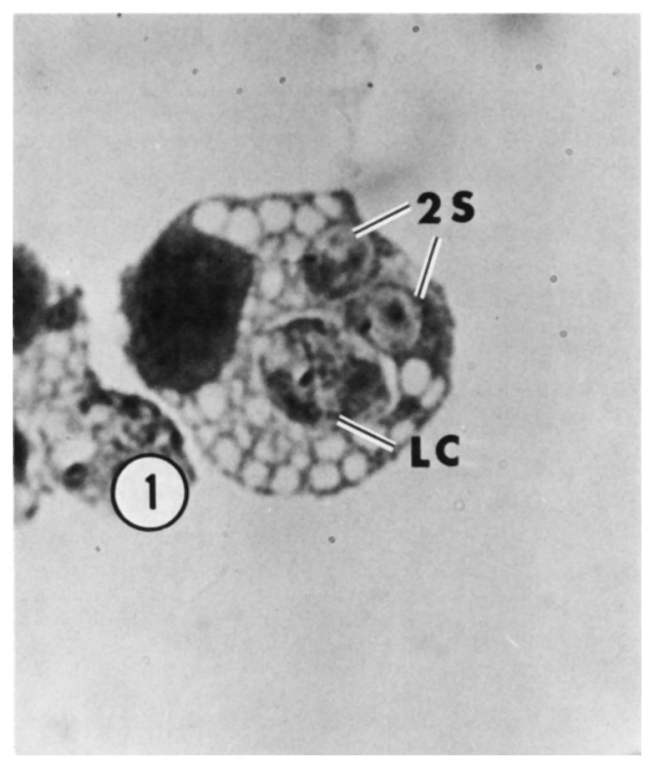

FIGURE 1. Typical macrophage in vitro infected with Leishmania donovani $2 \mathrm{~S}$ strain and subsequently with Leptomonas costoris (LC). Giemsa, $\times 1,200$. Differences in size and granulation are seen between the two species of parasites.

determine changes in parasite burden that were independent of the treatment. This allowed a 95\% probability with a $5 \%$ error of distinguishing an absolute difference of $15 \%$ between mortality rates caused by treatments, a difference that was considered to be significant.

\section{RESULTS}

A typical macrophage infected in vitro with both $L$. donovani ( $2 \mathrm{~S}$ strain and $L$. costoris) is shown in Figure 1. As digestion of the parasites proceeded, their structural integrity was lost, and parasites without well-defined nuclei, kinetoplasts (L. donovani), and bluestaining cytoplasmic granules (L. costoris) were counted as digested.

\section{Standardization of the bioassay}

Following washing, there was no detectable evidence for parasite division or extracellular multiplication during the course of the experiments. Standing cultures of $L$. donovani and L. costoris were initiated at the time of infection, incubated at $35 \mathrm{C}$ in CCM $5 \% \mathrm{CO}_{2}-95 \%$ air, without macrophages in Leighton tubes. For a typical case of $L$. donovani $2 \mathrm{~S}$ at $4 \mathrm{hr}$, there were $3.3 \pm 0.2 \times 10^{5}$ cells (mean $\pm \mathrm{SE}, n=4$ ) in $1 \mathrm{ml}$ per Leighton tube and at $30 \mathrm{hr}$ there were $3.0 \pm 0.1 \times 10^{5}$ cells $/ \mathrm{ml}(n=4)$. This represented a slight, but not significant, reduction in numbers for $L$. donovani $(t=1.43, P=0.1$ ). For standing cultures of $L$. costoris there were $1.19 \pm$ $0.01 \times 10^{6} \mathrm{cell} / \mathrm{ml}(n=4)$ at $4 \mathrm{hr}$ and $1.13 \pm$ $0.03 \times 10^{6}$ cells $/ \mathrm{ml}(n=4)$ after $28 \mathrm{hr}$, numbers that were not significantly different $(t=$ $0.374, P=0.91$ ). Cultures were more than $99 \%$ viable at $4 \mathrm{hr}$ and $95 \%$ viable at $28 \mathrm{hr}$ as tested by erythrosin B dye exclusion.

There was a persistent ingestion by the macrophages of parasites adherent to the cover glass, but this represented an exceedingly small error. Adherent $L$. donovani were counted $8 \mathrm{hr}$ (21 parasites/2,000 macrophages) and $24 \mathrm{hr}$ (13 parasites/2,000 macrophages) following infection. Adherent L. costoris were counted $4 \mathrm{hr}$ (22 parasites/2,000 macrophages) and $20 \mathrm{hr}$ (8 parasites/2,000 macrophages) following infection. There was no significant difference between species in the percent change in number of adherent parasites during this time interval $\left(\chi^{2}=0.97, P=\right.$ 0.32 ). Approximately five parasites were phagocytized per 1,000 macrophages between the two sample periods, because there was no significant change in standing culture cell numbers. Thus, the parasite burden in the final sample was overestimated by 0.005 . Subsequent data were not corrected for this minor amount.

There was no significant macrophage loss during the course of the experiment. For a typical experiment, following washing there were $1.64 \pm 0.09 \times 10^{5}$ macrophages $/ \mathrm{cm}^{2}$ (mean $\pm \mathrm{SE}, n=8$ ) and $15 \mathrm{hr}$ later there were $1.62 \pm 0.04 \times 10^{5}$ macrophages $/ \mathrm{cm}^{2}(t=0.116$, $P=0.95)$.

The time periods for macrophage exposure to the parasites and the ratio of parasites to macrophages were chosen for the following reasons besides being common in the literature. Ideally, we wished to examine a macrophage that has one, or occasionally two, $L$. donovani, and one or possibly two L. costoris so that the digestive capacity of the macrophage was not overtaxed and for ease of counting. A 4-hr exposure with a ratio of two $L$. donovani/adherent macrophage and one $L$. costoris/adherent macrophage provided this optimal infection. From initial experiments, macrophages exposed to $L$. costoris at a ratio of four parasites to one macrophage yielded 
TABLE I. Effect of L. donovani $2 S$ on macrophage digestion of subsequently ingested L. costoris. Data from five experiments; two replicates/treatment; 5,000 macrophages counted per entry in table.

\begin{tabular}{|c|c|c|c|c|}
\hline \multirow[b]{2}{*}{ Treatment } & \multicolumn{3}{|c|}{ Number of $L$. costoris per time postinfection } & \multirow[b]{2}{*}{$\%$ Change } \\
\hline & $4 \mathrm{hr}$ & $16 \mathrm{hr}$ & $28 \mathrm{hr}$ & \\
\hline \multicolumn{5}{|l|}{ 1. 12-hr change } \\
\hline CCM followed by LC infection & 2,235 & 741 & - & -66.8 \\
\hline $2 \mathrm{~S}$ followed by LC infection & 1,568 & 1,356 & - & $-13.5 P=0.01$ \\
\hline \multicolumn{5}{|l|}{ 2. 24-hr change } \\
\hline CCM followed by LC infection & 2,263 & - & 381 & -83 \\
\hline $2 S$ followed by LC infection & 1,394 & - & 597 & $-57 P=0.01$ \\
\hline
\end{tabular}

approximately one parasite/macrophage. Exposure to one parasite/macrophage yielded approximately 0.15 parasite/macrophage. Also, the parasite's distribution was described best by the Poisson distribution in the range of burden of parasites up to 0.5 and by the negative binomial from one and above. In the next $16 \mathrm{hr}$, approximately $85 \%$ of the parasites were cleared from the macrophages whether the parasite burden was 0.1 or 1 .

Of those macrophages still containing $L$. costoris, approximately $97 \%$ contained only one partially digested parasite. Thus, we concluded that the macrophage had the capacity to clear from one to three parasites equally. A ratio of one L. costoris per macrophage for 4hr infection resulted in a burden of about 0.2 per adherent macrophage, where approximately $86 \%$ of the infected cells had only one L. costoris and the remainder two parasites. For L. donovani, by choosing a ratio of two parasites/adherent macrophage and a 4 -hr infection, we obtained burdens of 0.5 to 0.7 , which followed a Poisson distribution. About $60 \%$ of the cells were infected and of these $78 \%$ had one parasite and $20 \%$ had two parasites.

These conditions then allowed us to examine usually a macrophage with one L. donovani and L. costoris. Because of a limited macrophage digestive capacity and the phenomenon of reticuloendothelial blockade, we did not wish infections where large numbers (more than two) of L. costoris or L. donovani were present within the macrophage.

\section{Bioassay results}

The sequential infection bioassay using the $2 S$ strain of $L$. donovani and $L$. costoris is shown in Tables I and II. After a slight initial depression at $20 \mathrm{hr}$, L. donovani $2 \mathrm{~S}$ increased
$50 \%$ during a 58 -hr period. This pattern was the same in macrophages infected with only $L$. donovani $2 \mathrm{~S}$ and in those infected with $L$. donovani $2 \mathrm{~S}$ and subsequently with $L$. costoris. Macrophages infected with only L. costoris cleared $67 \%$ of the parasites $16 \mathrm{hr}$ postinfection, $83 \%$ at $28 \mathrm{hr}$ postinfection, and $100 \%$ after $52 \mathrm{hr}$.

In contrast, macrophages initially infected with $L$. donovani $2 \mathrm{~S}$ and subsequently with L. costoris retained significantly more $L$. costoris than did controls. These doubly infected macrophages cleared only $14 \%$ of the L. costoris after $16 \mathrm{hr}$ postinfection, $57 \%$ after $28 \mathrm{hr}$, and $100 \%$ after $52 \mathrm{hr}$.

The simultaneous infection bioassay is shown in Table III. There was no significant difference in the ability of the macrophages to clear L. costoris, killed L. donovani $2 \mathrm{~S}$ with L. costoris, or killed L. donovani $\mathrm{Kh}$ with $L$. costoris $\left(\chi^{2}=3.6, P=0.17\right)$. In all cases, macrophages cleared about $80 \%$ of the L. costoris by $16 \mathrm{hr}$ postinfection. In contrast, macrophages coinfected with both $L$. donovani $2 \mathrm{~S}$ and L. costoris cleared only $56 \%$ of the L. costoris after $16 \mathrm{hr}$. This was significantly less than the controls.

The sequential bioassay using the Kh strain of $L$. donovani and L. costoris is shown in

TABLE II. Leishmania donovani $2 S$ and $K h$ growth in macrophages subsequently infected with L. costoris. Data from five experiments; $1,000(\mathrm{Kh})$ or $2,000(2 \mathrm{~S})$ infected macrophages were counted per entry in table.

\begin{tabular}{crrrrr}
\hline & \multicolumn{4}{c}{ Parasite number per time postinfection } & \\
\cline { 2 - 5 } Strain & \multicolumn{1}{r}{$8 \mathrm{hr}$} & $20 \mathrm{hr}$ & $34 \mathrm{hr}$ & $58 \mathrm{hr}$ & $\%$ Change \\
\hline 2S & 1,213 & 1,132 & 1,379 & 1,815 & +50 \\
$\mathrm{Kh}$ & 843 & 571 & 65 & 30 & -96.4 \\
\hline
\end{tabular}


TABLE III. Effect of L. donovani $2 S$ on macrophage digestion of $\mathrm{L}$. costoris during simultaneous infection. Data represent two replicates per treatment; 500 macrophages counted per entry in table.

\begin{tabular}{lccc}
\hline & \multicolumn{3}{c}{$\begin{array}{c}\text { Number of } \\
\text { L. costoris per } \\
\text { time postinfection }\end{array}$} \\
\cline { 2 - 3 } \multicolumn{1}{c}{ Treatment } & $4 \mathrm{hr}$ & $16 \mathrm{hr}$ & \% Change \\
\hline 1. Live LC & 537 & 108 & -80 \\
2. Killed 2S with live LC & 484 & 80 & -83 \\
3. Live 2S with live LC & 342 & 149 & $-56 P=0.01$ \\
4. Killed Kh with live LC & 440 & 98 & -78 \\
\hline
\end{tabular}

Tables II and IV. Leishmania donovani $\mathrm{Kh}$ did not persist in macrophages. After $58 \mathrm{hr}$, more than $96 \%$ were cleared. This pattern was the same in macrophages infected only with $L$. donovani $\mathrm{Kh}$ and subsequently with $L$. costoris. Macrophages infected only with $L$. costoris cleared $70 \%$ of the parasites $16 \mathrm{hr}$ postinfection. However, macrophages initially infected with $L$. donovani $\mathrm{Kh}$ and subsequently with $L$. costoris did not retain significantly more $L$. costoris than did the controls $\left(\chi^{2}=1.2, P=0.27\right)$. These macrophages cleared $61 \%$ of the L. costoris in $16 \mathrm{hr}$.

\section{DISCUSSION}

The major contribution of this paper is the demonstration that leishmanial parasites can alter the overall digestive functions of nonstimulated macrophages from nonimmune animals, and that the ability to effect such alterations is related to the protozoan strain's infectivity for the intact host. All cells used, both host and parasite, were eukaryotic and the bioassay system for macrophage digestive capacity used a parasite (L. costoris) closely related to the "test" organism ( $L$. donovani strains). The "test" organisms in these studies are obligate intracellular parasites in the mammalian host reticuloendothelial system. Thus, the results indicate that there are parasite attributes, differing among members of this family of organisms and among strains of the same species, which either enhance or diminish the ability of a particular species to survive phagocytosis, lysosomal fusion, and subsequently suppress host cell digestive capacity or alter other macrophage activities (Dwyer, 1979).

In some experiments, there was an apparent
TABLE IV. Effect of L. donovani $K h$ on macrophage digestion of subsequently ingested $\mathrm{L}$. costoris. Data are results of two experiments; two replicates per treatment; 1,000 macrophages were counted per entry in table.

\begin{tabular}{lccc}
\hline \multicolumn{1}{c}{ Treatment } & \multicolumn{2}{c}{$\begin{array}{c}\text { Number of } L \text {. costoris } \\
\text { per } \\
\text { time postinfection }\end{array}$} & \\
\cline { 2 - 3 } & $4 \mathrm{hr}$ & $16 \mathrm{hr}$ & $\%$ Change \\
\hline CCM followed by LC infection & 329 & 98 & -70 \\
Kh followed by LC infection & 310 & 110 & -61 \\
\hline
\end{tabular}

depression of $L$. donovani $2 S$ multiplication in macrophages infected with promastigotes. This has been noticed when amastigotes were used (Chang and Dwyer, 1976). Neither a depression of the number of $2 S$ parasites in a lag phase, an immediate increase in the number, nor a stationary lag phase of the number during the initial period of infection had an effect on the observed suppression of $L$. costoris digestion. However, in all of our experiments with the $2 S$ stock culture grown for 13 $\mathrm{yr}$ as a promastigote in Tanabe's medium, there has been variability with either a depressed lag, or stationary lag before growth or immediate growth. We can conclude only that this represents a parasite variability resulting from the time of harvest and a variability in the macrophages from different hamsters. The other possibility is cytotoxicity from the various batches of fetal calf serum when the parasites are resuspended in the CCM with $15 \%$ serum.

Although the results of our studies do not negate or counter previously expressed hypotheses that these parasites are resistant to lysosomal enzymes (Chang and Dwyer, 1976), they focus attention on the longer-term relationships between eukaryotic parasite and eukaryotic host cell. Moreover, they suggest that this relationship is manifest at the cellular level, perhaps apart from influences that the intact host may exert upon the cell-to-cell relationship through immune responses.

The exact nature of this relationship, however, remains unknown. In previous reports, it has been shown that not only do L. donovani promastigotes release a variety of species-specific macromolecules into incubation media, but that these parasites also exchange such molecules with their host cells (see Janovy, 1977, and references). In addi- 
tion, it is a well-established fact that culture forms of trypansomatid species differ metabolically in numerous ways (Janovy, 1977). Thus, infective parasites could suppress macrophage digestive capacity through an "active" process in which communicator molecules, inhibiting specific functions in host cells, are released by intracellular amastigotes. Conversely, the macrophage's digestive capacity, in the time following initial lysosomal fusion with the parasitophorous vacuole, could be inhibited "passively" through depletion of macrophage metabolic pools by the living intracellular amastigote. In the former case, infectivity could be the result of evolutionary adaptation by the parasite to the specific type of host cell. But in the latter case, infectivity perhaps could result from a fortuitous combination of specific parasite metabolic requirements and a host cell vulnerable to depletion of its intracellular metabolic pool. Other possibilities facilitating parasite survival have been mentioned by Dwyer (1979) and Trager (1974).

The demonstration that there are parasite species and strain-specific attributes that function to control the relationship between obligate intracellular parasite and nonstimulated, nonimmune phagocytic cells in vitro, suggests that further studies of this relationship at the cellular level might focus on the two hypotheses we have described.

\section{ACKNOWLEDGMENT}

This work was supported in part by NSF grant PCM 75-23628.

\section{LITERATURE CITED}

AleXander, J., AND K. Vickerman. 1975. Fusion of host cell secondary lysosomes with the parasitophorous vacuoles of Leishmania mexicana-infected macrophages. J. Protozool. 22: $502-508$.

Chang, K. P., And D. M. Dwyer. 1976. Multiplication of a human parasite (Leishmania donovani) in phagolysosomes of hamster macrophages in vitro. Science 193: 678-680.

— AND - 1978. Leishmania donovani. Hamster macrophage interactions in vitro: Cell entry, intracellular survival and multiplication of amastigotes. J. Exp. Med. 147: 515-530.

DAgGetT, P. M., J. E. DECKER, AND J. JANOVY, JR. 1978. Some physiological alterations accompanying infectivity to mammals by four genera of trypanosomatidae. Comp. Biochem. Physiol. 59A: $363-366$.

Dollahon, N. R., AND J. JANOVY, JR. 1971. Insect flagellates from feces and gut contents of four genera of lizards. J. Parasitol. 57: 1130-1132.

DWYER, D. M. 1979. Membrane interactions between Leishmania and host cells. In Microbiology-1979, D. Schlessinger (ed.). ASM. pp. $130-134$.

JANOVY, J., JR. 1967. Respiratory changes accompanying leishmania to leptomonad transformation in Leishmania donovani. Exp. Parasitol. 20: $51-55$.

- 1977. Problems in the comparative physiology of some trypanosomatid flagellates. Acta Trop. 34: 177-184.

STAUBER, L. A. 1966. Characterization of strains of Leishmania donovani. Exp. Parasitol. 18: 111.

Trager, W. 1974. Some aspects of intracellular parasitism. Science 183: 269-273.

Wallace, F. G., S. R. Todd, AND W. J. Rogers. 1965. Flagellate parasites of water striders with descriptions of Leptomonas costoris n. sp. J. Protozool. 12: 390-393.

WASLEY, G. D., AND R. JOHN. 1972. Cultivation of mammalian macrophages. In Animal tissue culture advances in techniques, G. D. Wasley (ed.). Butterworths, London, Ch. 5, p. 161. 